\title{
Comparative Efficacy of Fexofenadine Versus Levocetrizine Versus Desloratadine via 1\% Histamine Wheal Suppression
} Test

\author{
KC S, Aryal A, Adhikary M, Karn D
}

\author{
Department of Dermatology \\ Kathmandu University School of Medical Sciences \\ Dhulikhel, Kavre, Nepal.
}

\section{Corresponding Author}

Shekhar KC

Department of Dermatology,

Kathmandu University School of Medical Sciences, Dhulikhel, Kavre, Nepal.

E-mail: drshekharkc@gmail.com

\section{Citation}

KC S, Aryal A, Adhikary M, Karn D. Comparative Efficacy of Fexofenadine Versus Levocetrizine Versus Desloratadine via $1 \%$ Histamine Wheal Suppression Test. Kathmandu Univ Med J. 2020;69(1):19-22.

\begin{abstract}
Background

Urticaria and allergic dermatoses remains a great challenge to treating dermatologist. Histamine is the major mediator in such disorders. Antihistamines as levocetrizine, fexofenadine and desloratadine are often used to treat such conditions. Ability of antihistamines to suppress the allergic response helps to evaluate the efficacy of the medicine.
\end{abstract}

\section{Objective}

To compare the efficacy of levocetrizine versus fexofenadine versus desloratadine in suppressing histamine induced wheals in adults.

\section{Method}

One hundred and two healthy adult volunteers completed the study. Subjects were randomized into 3 groups using an envelope method. First group received fexofenadine ( $N=36)$, second group received levocetrizine $(N=37)$, and third group received desloratadine $(\mathrm{N}=29)$. Pretesting was performed by skin prick test with histamine $1 \%$ (positive control) and normal saline (negative control). Wheal size was recorded before and after the treatment (at 0.5, 1, 2, 4 and 24 hours).

\section{Result}

At 30 minutes and 1 hour fexofenadine showed statistically significant wheal suppression than levocetrizine and desloratadine $(p=0.0016)$. However by 2 and 4 hours all three antihistamines; fexofenadine, levocetirizine and desloratadine showed significant suppression of wheal. Whereas at 24 hours desloratadine showed greater wheal suppression than levocetrizine and fexofenadine $(p=0.014)$.

\section{Conclusion}

The results of the present study showed that fexofenadine presented early onset of action but longer suppression of wheal size was seen with desloratadine as compared to other antihistamines. These potentials could be employed in clinical aspects; depending upon the response needed.

\section{KEY WORDS}

Desloratadine, Fexofenadine, Levocetrizine 


\section{INTRODUCTION}

Histamine is the major mediator in allergic reactions. ${ }^{1,2}$ Urticaria significantly impairs the quality of life and is difficult to treat in many instances. Antihistamine is used to treat such condition as an initial choice. With rising varieties of $\mathrm{H} 1$-antihistamine, from first generation to second generation, clinician needs to pick the best and effective H1-antihistamine. There are different methods to compare the clinical efficacy of antihistamines. The best method is to compare the clinical efficacy by administering $\mathrm{H} 1$-antihistamine for chronic spontaneous urticaria and monitor the clinical outcome; but it can be extremely expensive, time consuming and can have ethical and logistic issues. ${ }^{3,4}$ Also study on determination of in-vitro potency (ki) of antihistamines to predict clinical efficacy of $\mathrm{H} 1$-antihistamine showed huge differences in volume of distribution and tissue accumulation in humans hence this method is not regarded as a good forecaster of clinical efficacy. However, histaminergic wheal and flare model appear to be the best indicator to evaluate the clinical efficacy of antihistamine. ${ }^{3}$ Non-sedating second generation antihistamines are recommended as first line treatment for urticaria. ${ }^{5}$ However the choice depends upon the judgement of treating physician and the patients status. We have here conducted a comparative study to compare the efficacy of three second generation antihistamines by their ability to suppress the histamine induced wheal in healthy subjects.

\section{METHODS}

This is prospective, randomized, non-blinded, clinical trial conducted from January - March 2018 with a prior approval from institutional review board (IRB Protocol approval no. 89/14). A total of 150 healthy adult male and female volunteers between age of 20 to 30 were included in the study. Altogether 48 volunteers lost to follow up and only 102 volunteers completed the study. Pregnancy, lactation, active dermographism, angry back syndrome, atopy, drug hypersensitivity, presence of current active allergic disease like asthma, urticaria and upper respiratory tract infection, volunteers who were taking antihistamines for any reason, steroids or immunosuppressant for last 2 weeks were excluded from the study.

The volunteers were randomized by envelope method into 3 groups. Group A received fexofenadine $180 \mathrm{mg}$, group $B$ received levocetrizine $5 \mathrm{mg}$, and group $C$ received $5 \mathrm{mg}$ desloratadine in empty stomach with one glass of water. The test was performed in a marked square area of $1.5 \mathrm{x}$ $1.5 \mathrm{~cm}$ at different sites each time, on the flexor aspect of the forearm (right and left). A drop of $1 \% \mathrm{w} / \mathrm{v}$ of histamine solution (positive control) and $0.9 \%$ normal saline (negative control) was placed on the flexor aspect of the forearm. The skin was pricked through the histamine solution and normal saline with a lancet. After 15 minutes, baseline wheal size was estimated by measuring mean of largest diameter of the wheal and diameter perpendicular to this, with the help of transparent ruler.

Similar skin prick test with histamine were performed at 30 minutes, 1 hour, 2 hours, 4 hours and 24 hours respectively post medication at different sites of forearm in each individual and respective wheal diameter was measured after 15 mins of each prick.

For all continuous variables arithmetic mean, standard deviation and range were calculated. For categorical variables, frequencies were calculated. Student's T-test was used to evaluate the statistical significance of differences observed between groups for continuous variables. A $5 \%$ margin of error ( $p$-value $<0.05$ ) was considered to be significant statistically. For all statistical analyses, the Statistical Package for Social Sciences (SPSS) version 16.0 statistical software package (SPSS Inc, Chicago, IL, USA) was used.

\section{RESULTS}

A total of 49 male and 53 female volunteers completed the study. Table 1 represents the basic demography of volunteers in three different groups.

Table 1. Volunteers demography

\begin{tabular}{|llll} 
& GROUP A & GROUP B & GROUP C \\
\hline Age (years) & $25 \pm 2.94$ & $24.02 \pm 2.53$ & $24.72 \pm 1.99$ \\
Gender & & & \\
\hline Male & 17 & 18 & 14 \\
\hline Female & 19 & 19 & 15 \\
\hline
\end{tabular}

Table 2. The mean values of wheal sizes in response to histamine challenge for fexofenadine $180 \mathrm{mg}$, levocetirizine $5 \mathrm{mg}$, and desloratadine $5 \mathrm{mg}$ before and after $1 / 2,1,2,4,24$ hours following drug administration

\begin{tabular}{llllllll|}
\hline Medicine provided & \multicolumn{7}{l}{ Mean wheal Size in $\mathrm{mm}$} \\
& Pretest & $1 / 2 \mathrm{~h}$ & $1 \mathrm{~h}$ & $2 \mathrm{~h}$ & $4 \mathrm{~h}$ & $24 \mathrm{~h}$ \\
\hline Fexofenadine & 7.66 & 6.27 & 5.21 & 3.90 & 3.40 & 6.98 \\
\hline Levocterizine & 8.23 & 7.46 & 6.88 & 4.84 & 4.13 & 6.76 \\
\hline Desloratadine & 6.90 & 6.17 & 5.72 & 3.86 & 3.32 & 4.67
\end{tabular}

For wheal suppression, measured as absolute change from baseline, fexofenadine $180 \mathrm{mg}$ daily was significantly more effective than levocetrizine and desloratadine from 30 minutes to 1 hours ( $p=0.043$ and $p=0.0016$ respectively)

Table 3. Comparison of mean \pm standard deviation of baseline (predose) wheal size and 30 minutes wheal size

\begin{tabular}{llll} 
& Baseline $(\mathbf{m m})$ & $\mathbf{3 0}$ minutes $(\mathbf{m m})$ & P-value \\
\hline Fexofenadine & $7.66 \pm 2.92$ & $6.27 \pm 2.81$ & 0.043 \\
Levocetrizine & $8.23 \pm 3.19$ & $7.46 \pm 0.35$ & 0.22 \\
Desloratadine & $6.90 \pm 3.72$ & $6.17 \pm 3.32$ & 0.43
\end{tabular}


Table 4. Comparison of mean \pm standard deviation of baseline (predose) wheal size and 1 hour wheal size

\begin{tabular}{|llll} 
& Baseline $(\mathrm{mm})$ & $\mathbf{1}$ hour $(\mathrm{mm})$ & P-value \\
\hline Fexofenadine & $7.66 \pm 2.92$ & $5.21 \pm 3.38$ & 0.0016 \\
\hline Levocetrizine & $8.23 \pm 3.19$ & $6.88 \pm 3.56$ & 0.09 \\
\hline Desloratadine & $6.90 \pm 3.72$ & $5.72 \pm 2.45$ & 0.15 \\
\hline
\end{tabular}

Table 5. Comparison of mean \pm standard deviation of baseline (predose) wheal size and 2 hours wheal size

\begin{tabular}{|llll|} 
& Baseline $(\mathrm{mm})$ & $\mathbf{2}$ hours $(\mathrm{mm})$ & P-value \\
\hline Fexofenadine & $7.66 \pm 2.92$ & $3.90 \pm 2.84$ & 0.000 \\
\hline Levocetrizine & $8.23 \pm 3.19$ & $4.84 \pm 3.78$ & 0.000 \\
\hline Desloratadine & $6.90 \pm 3.72$ & $3.86 \pm 2.63$ & 0.000 \\
\hline
\end{tabular}

At 2 hours and 4 hours all three antihistamines fexofenadine $180 \mathrm{mg}$, levocetrizine 5mg, and desloratadine $5 \mathrm{mg}$ significantly suppressed the wheal size when compared to baseline wheal size. Hence the maximum activity of fexofenadine, levocetrizine, and desloratadine was seen at 2 hours and 4 hours.

Table 6. Comparison of mean \pm standard deviation of baseline (predose) wheal size and 4 hours wheal size

\begin{tabular}{|llll} 
& Baseline $(\mathrm{mm})$ & $\mathbf{4}$ hours $(\mathrm{mm})$ & P-value \\
\hline Fexofenadine & $7.66 \pm 2.92$ & $3.40 \pm 2.63$ & 0.000 \\
\hline Levocetrizine & $8.23 \pm 3.19$ & $4.13 \pm 3.38$ & 0.000 \\
\hline Desloratadine & $6.90 \pm 3.72$ & $3.32 \pm 2.82$ & 0.000
\end{tabular}

At 24 hours all three antihistamines showed suppression of wheal size, but statistically significant suppression of wheal size was seen only with desloratadine. Hence desloratadine showed more effectiveness through 24 hours as compared with fexofenadine and levocetrizine in suppressing the histamine wheal suppression test.

Table 7. Comparison of mean \pm standard deviation of baseline (predose) wheal size and 24 hours wheal size

\begin{tabular}{|llll} 
& Baseline $(\mathbf{m m})$ & $\mathbf{2 4}$ hours $(\mathbf{m m})$ & P-value \\
\hline Fexofenadine & $7.66 \pm 2.92$ & $6.98 \pm 3.87$ & 0.40 \\
Levocetrizine & $8.23 \pm 3.19$ & $6.76 \pm 3.81$ & 0.07 \\
Desloratadine & $6.90 \pm 3.72$ & $4.67 \pm 2.93$ & 0.014
\end{tabular}

\section{DISCUSSION}

With the growing figures of antihistamines, much attention has been set to compare the relative efficacies of the various compounds. Among various clinical and laboratory tests, the most common tool used for the comparison of efficacy involves an epicutaneous injection of histamine. ${ }^{6}$ It helps to assess the ability of systemically introduced antihistamine to inhibit the iatrogenic histamine induced wheal and flare response.
Histamine induced wheal and flare suppression model is a very practical and useful modality for the objective assessment of pharmacodynamic properties of $\mathrm{H} 1$-antihistamines. ${ }^{7,8}$ The sensitivity, specificity, and reproducibility of this in-vivo test is considered to be excellent when it is executed in a standardized manner. ${ }^{6,7}$

Analysis of the results in the present study showed that fexofenadine significantly reduced the wheal size at 30 minute and 1 hour $(p=0.04$ and $p=0.001)$ when compared with levocetrizine and desloratadine. In the present study fexofenadine has early onset of action. However, fexofenadine didn't significantly suppress wheal size at 24 hours of drug intake. A similar study appraisal by Simons FE et al. revealed that fexofenadine had a significantly faster onset of action than loratadine in suppressing histaminergic wheal. $^{8}$

All the tested medicines, fexofenadine, levocetrizine, and desloratadine are second generation antihistamines. ${ }^{9}$ Fexofenadine is an active metabolite of terfenadine. ${ }^{9,10}$ Fexofenadine has a peak plasma levels at 1-3 $\mathrm{h}$ after administration and an elimination half-life of $11-15$ h. ${ }^{9}$ The duration of action of antihistamine is determined by the time for the wheal to remain inhibited by at least $70 \%$. Fexofenadine $180 \mathrm{mg}$ and cetirizine $10 \mathrm{mg}$ had $70 \%$ of wheal inhibition period of 9.3 hours and 19 hours respectively. ${ }^{11}$ The shorter duration of action of fexofenadine is due to its active secretion into the intestine and urine by P-glycoprotein pathway. ${ }^{12}$

A study by Dhanya et al. demonstrated that fexofenadine has early onset of action with significant suppression of wheal size at half an hour of drug intake. ${ }^{10}$ The same study revealed that levocetirizine had maximum response after three and six hours whereas desloratadine had superior inhibition at the end of $24 \mathrm{~h}$ when compared to levocetirizine and fexofenadine.

A randomized, placebo-controlled, double-blind, trial conducted by Meltzer et al. showed that fexofenadine has early onset of action at 1 hour and is superior to desloratadine in suppressing histamine induced wheals throughout 24 hours. ${ }^{13}$ But in our study, desloratadine seemed superior to fexofenadine for suppressing the wheal at 24 hours.

Levocetirizine is an active enantiomer of cetirizine with an onset of action at about one hour and an elimination halflife of 11 hours. ${ }^{9}$ In the present study, levocetrizine showed statistically significant wheal suppression at 2 hours and 4 hours. But levocetrizine failed to continue the significant wheal suppression at 24 hours.

A study conducted by Grant et. al. for comparison of different anthistamines showed that levocetirizine and fexofenadine inhibited the wheal response after 1 hour; and reached their peak inhibition after 4 hours. ${ }^{1}$ In the same study, levocetirizine was seen as the most potent and consistently effective drug for inhibiting the histamine- 
induced wheal surface area when compared with other antihistamines whereas, loratadine was the least potent one. ${ }^{1}$

Devalia et al. revealed a dramatic inhibition of the wheal response beginning within 1 hour after dosing with levocetrizine which lasted for 28 hours. ${ }^{14}$ In contrast our study failed to show a statistically significant wheal suppression lasting for 24 hours with levocetrizine. A similar study showed that $1.25 \mathrm{mg}$ levocetirizine was statistically more effective than $10 \mathrm{mg}$ desloratadine in suppressing histamine induced wheal at 4 hours of dosing. ${ }^{15}$ The present study revealed that both the drugs significantly suppressed wheal at 2 and 4 hours but only desloratadine could significantly suppress wheal for an extended time.

Desloratadine is a biologically active metabolite of loratadine with potent non-sedating, long-acting, and selective peripheral $\mathrm{H} 1$-receptor antagonistic activity. ${ }^{9,10,16-18}$ It has peak plasma level at 2-3 hours and half-life of 21$24 \mathrm{~h}$, permitting once-daily dosing. ${ }^{16,17}$ Many studies have shown that desloratadine is less efficacious in suppressing in-vivo histamine induced cutaneous response however, it display prolong duration of action. ${ }^{10,12,13,18}$

In the current study, wheal suppression was significantly high at 2 hours, 4 hours and 24 hours after taking single dose of desloratadine $10 \mathrm{mg}$. Desloratadine was the only antihistamine demonstrating suppression of the wheal size significantly for 24 hours period. It may be related to elimination the half-life of fexofenadine and levocetrizine being shorter than that of desloratadine owing to their shorter duration of action. No any serious adverse clinical events were recorded during this study period.

\section{CONCLUSION}

The present study revealed that fexofenadine has an early onset of action but longer suppression of wheal size was seen with desloratadine. These potentials could be employed in clinical aspects depending upon the response needed.

\section{REFERENCES}

1. Grant JA, Riethuisen JM, Moulaert B, et. al. A double-blind, randomized, single-dose, crossover comparison of levocetirizine with ebastine, fexofenadine, loratadine, mizolastine, and placebo: suppression of histamine-induced wheal-and-flare response during 24 hours in healthy male subjects. Annals of Allergy, Asthma and Immunology. 2002;1;88(2):190-7.

2. Devillier P, Bousquet J. Inhibition of the histamine-induced weal and flare response: a valid surrogate measure for antihistamine clinical efficacy?. Clinical \& Experimental Allergy. 2007 Mar;37(3):400-14.

3. Church MK, Maurer M. H1-antihistamines and urticaria: how can we predict the best drug for our patient?. Clinical \& Experimental Allergy. 2012;42(10):1423-9.

4. Schoepke N, Church MK, Maurer M. The Inhibition by Levocetirizine and Fexofenadine of the Histamine induced Wheal and Flare Response in Healthy Caucasian and Japanese Volunteers. Acta Derm Venereol. 2013; 93: 286-93.

5. Zuberbier T, Aberer W, Asero R, et. al. The EAACI/GA 2 LEN/EDF/ WAO Guideline for the definition, classification, diagnosis, and management of urticaria: the 2013 revision and update. Allergy. 2014;69(7):868-87.

6. Monroe EW, Daly AF, Shalhoub RF. Appraisal of the validity of histamine-induced wheal and flare to predict the clinical efficacy of antihistamines. J Allergy Clin Immunol. 1997;99:798-806.

7. Simons FER, McMillan JL, Simons KJ. A double-blind, singledose, crossover comparison of cetirizine, terfenadine, loratadine, astemizole, and chlorpheniramine versus placebo: suppressive effects on histamineinduced wheals and flares during 24 hours in normal subjects. J Allergy Clin Immunol. 1990;86:540-7.

8. Simons FE, Simons KJ. Peripheral H1-blockade effect of fexofenadine. Annals of Allergy, Asthma and Immunology. 1997;1;79(6):530-2.

10. Dhanya NB, Thasleem Z, Rai R, et. al. Comparative efficacy of levocetirizine, desloratidine and fexofenadine by histamine wheal suppression test. Indian J Dermatol Venereol Leprol. 2008;74:361-3

11. Purohit A, Duvernelle C, Melac M, et. al. Twenty-four hours of activity of cetirizine and fexofenadine in the skin. Ann Allergy Asthma Immunol. 2001;4(4):387-92.

12. Church DS, Church MK. Pharmacology of antihistamines. World Allergy Organization Journal. 2011 Dec;4(3):S22.

13. Meltzer EO, Gillman SA. Efficacy of fexofenadine versus desloratadine in suppressing histamine-induced wheal and flare. Allergy Asthma Proc. 2007;28:67-73.

14. Devalia JL, DeVos C, Hanotte F, et. al. A randomized, double-blind, crossover comparison among cetirizine, levocetirizine, and ucb 28557 on histamine-induced cutaneous responses in healthy adult volunteers. Allergy. 2001 Jan;56(1):50-7.

15. Popov TA, Dumitrascu D, Bachvarova A, et al. A comparison of levocetirizine and desloratadine in the histamine-induced wheal and flare response in human skin in vivo. Inflammation Research. 2006 Jun 1;55(6):241-4.

16. Henz BM. The pharmacologic profile of desloratadine: a review. Allergy. $2001 \mathrm{Mar} ; 56: 7-13$.

17. Ring J, Hein R, Gauger A, et. al. Desloratadine Study Group. Once-daily desloratadine improves the signs and symptoms of chronic idiopathic urticaria: a randomized, double-blind, placebo-controlled study. International journal of dermatology. 2001 Jan;40(1):72-6.

18. Purohit A, Melac M, Pauli G, et. al. Comparative activity of cetirizine and desloratadine on histamine-induced wheal-and-flare responses during 24 hours. Annals of Allergy, Asthma and Immunology. 2004 Jun 1;92(6):635-40. 